\title{
MUC1 gene polymorphism rs4072037 and susceptibility to gastric cancer: a meta-analysis
}

\author{
Xinyang Liu ${ }^{1 \dagger}$, Zhichao Wang ${ }^{2 \dagger}$, Xiaowei Zhang ${ }^{1 \dagger}$, Jinjia Chang ${ }^{1 \dagger}$, Wenbo Tang ${ }^{1}$, Lu Gan ${ }^{1}$, Zheng Wu ${ }^{1}$ and Jin Li ${ }^{i^{*}}$
}

\begin{abstract}
The association between MUC1 polymorphism rs4072037 and the risk of gastric cancer has been described in several studies. However, these studies yielded inconsistent results, especially in different pathological type of gastric cancer. Therefore, we performed this meta-analysis to evaluate the relationship between MUC1 gene polymorphism and gastric cancer susceptibility. A comprehensive database search was performed to identify eligible studies. Odds ratios with 95\% confidence intervals were calculated to assess the strength of the association between MUC1 rs4072037 and risk of gastric cancer. Subgroup analyses, publication bias, and sensitivity analyses were also conducted. PubMed, EMBASE, Web of Science and CNKI databases were systematically searched to identify relevant studies. A total of 9 studies (12 datasets) were included in the meta-analysis including 10,410 cases and 11,437 controls. Overall, the $G$ allele at rs4072037 of MUC1 gene was associated with a significant decreased gastric cancer risk (OR=0.70, 95\% Cl: 0.64-0.76). The association was significant in both anatomic location and pathological subtype subgroup analyses. However, the association was detected in Asian rather than Caucasian. Our findings demonstrate that the presence of the $\mathrm{G}$ allele at rs4072037 of the MUC1 gene may contribute to protection against gastric cancer in Asian. Further large studies of multiethnic groups are needed to validate these findings.
\end{abstract}

Keywords: MUC1; Polymorphism; Genetic; Stomach neoplasms; Meta-analysis

\section{Introduction}

Gastric cancer (GC) is the fourth most common cancer and the second leading course of cancer-related deaths worldwide, with an estimated 989,600 new cases and 738,000 deaths in 2008 (Jemal et al. 2011). Although the incidence and mortality of GC have decreased over the past few decades, it is still a heavy burden in developing areas like East Asia, Eastern Europe, and South America. The etiology of GC remains unclear, although environmental risk factors and host genetic factors are both thought to play a role in the GC carcinogenesis (Wu et al. 2005). Helicobacter pylori is the single most important etiological agent in the pathogenesis of GC (Blaser 2000), and it is becoming increasingly clear that several specific host genes are involved in response to H.pylori colonization, immune escape and mucosal injury in the stomach.

\footnotetext{
* Correspondence: fudanlijin@163.com

${ }^{\dagger}$ Equal contributors

'Department of Medical Oncology, Fudan University Shanghai Cancer Center; Department of Oncology, Shanghai Medical College, Fudan University, Shanghai 200032, PR China

Full list of author information is available at the end of the article
}

MUC1 gene is a member of the mucin family. It encodes a membrane-bound glycoprotein, which functions in protection of epithelial surfaces against environmental insults (Gendler 2001). For example, it can block the adhesion of H.pylori blood group antigen-binding adhe$\sin (\mathrm{BabA})$ and sialic acid-binding adhesin (SabA) to the gastric mucosa, thus limiting H.pylori colonization (Linden et al. 2009; Skoog et al. 2012). The association between $M U C 1$ polymorphism rs4072037 and the risk of gastric cancer has been described in previous studies (Xu et al. 2009; Jia et al. 2010) with a candidate gene approach. Recently, a genome-wide association study (GWAS) (Abnet et al. 2010) performed in Chinese population identified the same suspicious locus in the scanning phase but not in the second phase. After combining the two phases, it still failed to reach genome-wide significance. Similarly, case-control study in Japan demonstrated that rs4072037 was only associated with diffusetype gastric cancer but not intestinal-type gastric cancer. Since then, several studies were performed to validate these findings. Although the majority of the results are similar, inconsistency exists regarding to the role of 
rs4072037 in different pathological types of gastric cancer and different ethnic groups. A meta-analysis has been conducted by Zheng et al. (Zheng et al. 2013). However, their meta-analysis has some obvious limitations. Therefore, we performed this meta-analysis to evaluate the relationship between MUC1 gene polymorphism at rs4072037 and gastric cancer susceptibility and assess the effect size of the association in order to clarify the inconsistency among published studies.

\section{Materials and methods}

\section{Literature search strategy}

We performed a systematic review and meta-analysis on $M U C 1$ gene polymorphisms and gastric cancer susceptibility in accordance with the PRISMA Statement (Preferred Reporting Items for Systematic Reviews and Meta-Analyses) (Liberati et al. 2009) (Additional file 1). We searched MEDLINE (PubMed), EMBASE, Web of Science, and CNKI (China National Knowledge Infrastructure) without language restrictions to December 12, 2013, using the following search algorithms: ("MUC1" OR "mucin1" OR "1q22") AND ("polymorphism" OR "SNP") AND ("gastric neoplasms" OR "gastric cancer" OR "gastric carcinoma"). In addition, reference lists of identified studies and related GWAS were also hand-searched to identify potential eligible studies.

\section{Selection criteria}

Study eligibility was determined independently by two reviewers (LXY and ZXW). Disagreements were solved by consensus. Studies were considered for inclusion if they meet the following criteria: (i) evaluated MUC1 gene polymorphism rs4072037 and gastric cancer susceptibility, (ii) were cohort-based or case-control studies, and (iii) reported data necessary to calculate the odds ratios (ORs) with corresponding 95\% confidence intervals (95\% CIs). If such data were unavailable, attempts were made to contact the first author and/or corresponding author via e-mail to provide the missing data before the study was excluded from the final analysis. The major exclusion criteria included: (i) reviews, case-only studies, or familial studies, (ii) lack of sufficient data for calculation of ORs with $95 \%$ CIs, and (iii) duplication of previous publications or replicated samples.

\section{Data extraction and quality assessment}

Data extraction was carried out independently by two reviewers (LXY and ZXW) using a pre-defined standard form. Disagreements were resolved by discussion with a third author (WZC). From each study, the following information was extracted: first author's name, year of publication, ethnicity of the patients, source of control groups (population- or hospital-based controls), age, sex, genotyping method, Hardy-Weinberg equilibrium (HWE) of controls, and frequency of various genotypes in cases and controls, adjusted ORs and 95\% CIs. If allele frequencies were not given, they were calculated from the corresponding genotype distributions. If genotype frequencies were not given, they were calculated from allele frequencies only when the study was in accordance with HWE. For one study (Saeki et al. 2011), which included datasets of different ethnic populations and reported the results separately, the data were collected separately and the datasets were recognized as independent studies. Study quality was assessed independently by LXY and ZXW using the 10-point scoring scale for quality of genetic association studies proposed by Clark and Baudouin (Clark and Baudouin 2006).

\section{Statistical analysis}

ORs and 95\% CIs were used to assess the strength of the associations between MUC1 polymorphism rs4074037 and gastric cancer risk. OR represents the odds that a certain genotype occurring in gastric cancer patients, compared to the odds of the genotype occurring in controls. A $P$ value of $<0.05$ in a Z-test indicated statistical significance for the associations. Adjusted ORs and 95\% CIs were used if reported. Otherwise, the pooled ORs and $95 \%$ CIs without adjustments were calculated for the following genotypic models: allele (G vs. A), homozygote (GG vs. AA), heterozygote (AG vs. AA), dominant (GG/AG vs. AA), and recessive (GG vs. AG/AA). If adjusted ORs and 95\% CIs were reported based on A allele vs. $G$ allele, they were converted to $G$ allele vs. A allele.

A Cochrane chi-square-based Q-test was performed to test the heterogeneity among studies or cohorts. The $I^{2}$ tests were performed to assess the statistical heterogeneity, and the Q-statistic tests with $P<0.10$ were used to define a significant degree of heterogeneity. A fixedeffect model was used when there was no heterogeneity ( $P \geq 0.10$ for Q-test) (Mantel and Haenszel 1959), otherwise a random-effect model was used (DerSimonian and Laird 1986). We also estimated the statistical power of each individual study as determined by the probability of detecting a true association between MUC1 polymorphism rs4072037 and gastric cancer risk at the 0.05 level of significance. The estimation was based on the method described earlier (Schlesselman 1982).

For exploration of heterogeneity, subgroup analyses were performed based on anatomic location, pathological subtype, ethnicity, source of control, sample size $(\leq 1000$ and $>1000$ subjects), quality scores (score $>7$ or $\leq 7$ ) adjustment and statistical power. Sensitivity analyses were performed by deletion of a single study each time to reflect the influence of the individual data set on the pooled ORs. Begg's funnel plots (Egger et al. 1997) were used to assess the publication bias. Statistical analyses were conducted 
using Review manager Version 5.1 (Copenhagen, The Nordic Cochrane Centre, The Cochrane Collaboration, 2011).

\section{Results}

\section{Characteristics of the studies}

The process of selection of studies in the meta-analysis is summarized in a flow diagram (Figure 1). Database search revealed 99 potentially relevant publications. Eventually, 9 studies (Xu et al. 2009; Jia et al. 2010; Abnet et al. 2010; Yang et al. 2012; Palmer et al. 2012; Zhang et al. 2011; Saeki et al. 2011; Li et al. 2013; Song et al. 2013) that consisted of 12 datasets were eligible based on the inclusion/ exclusion criteria. The main characteristics of included studies are shown in a table (Additional file 2).

\section{Overall effects for meta-analysis}

All the 12 datasets including 10,410 cases and 11,437 controls presented enough data on the allele model. Two studies did not report the frequencies of each genotype and HWE. Of them, one study (Saeki et al. 2011) (including 4 datasets) only reported adjusted ORs and corresponding 95\% CIs under the allele model, and the other study (Abnet et al. 2010) reported adjusted ORs and corresponding 95\% CIs under allele, homozygote and heterozygote model. Thus, they were not included in the meta-analyses under some of the models.

In the overall analysis, we detected a significant association between the $\mathrm{G}$ allele at rs4072037 with decreased gastric cancer risk under the allele model ( $G$ vs. A; $\mathrm{OR}=0.70$, 95\% CI: 0.64-0.76; 10,410 cases and 11,437 controls.) (Table 1, Figure 2), homozygote model (GG vs. AA; $\mathrm{OR}=0.63$, 95\% CI: $0.52-0.76 ; 8,843$ cases and 8,341 controls), heterozygote model (AG vs. AA; OR=0.68, 95\% CI: 0.63-0.73; 8,843 cases and 8,341 controls), dominant model (GG/AG vs. AA; OR=0.65, 95\% CI: 0.54-0.77; 6,209 cases and 5,039 controls), and recessive model (GG vs. AG/AA; OR=0.76, 95\% CI: 0.63-0.92; 6,209 cases and 5,039 controls). Significant heterogeneity was detected in allele and dominant models among studies.

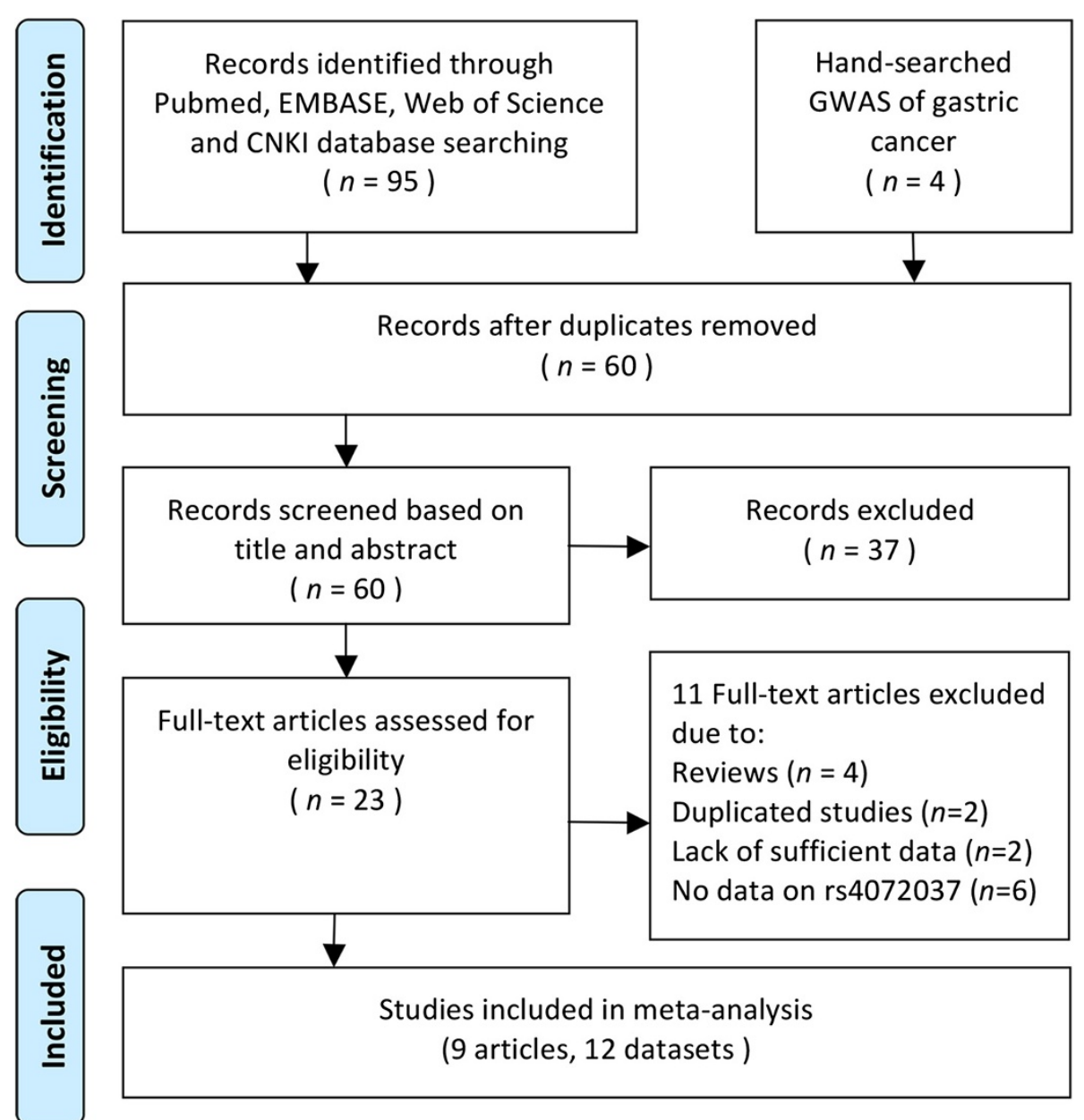

Figure 1 Flow diagram of the study process. CNKI, China National Knowledge Infrastructure; GWAS, genome-wide association studies. 
Table 1 Meta-analyses of the association between MUC1 polymorphism rs4072037 and gastric cancer risk under alternative models

\begin{tabular}{llllllccc}
\hline Comparison & No. of datasets & Case & Control & OR $(\mathbf{9 5} \% \mathbf{C l})$ & $\boldsymbol{P}$ for OR & $\boldsymbol{P}$ for heterogeneity & $\mathbf{I}^{\mathbf{2}}$ (\%) & Model \\
\hline G vs. A & 12 & 10410 & 11437 & $0.70(0.64,0.76)$ & $<0.00001$ & 0.04 & 47 & Random \\
GG vs. AA & 8 & 8438 & 8341 & $0.63(0.52,0.76)$ & $<0.00001$ & 0.47 & 0 & Fixed \\
AG vs. AA & 8 & 8438 & 8341 & $0.68(0.63,073)$ & $<0.00001$ & 0.28 & 19 & Fixed \\
GG/AG vs. AA & 7 & 6209 & 5039 & $0.65(0.54,0.77)$ & $<0.00001$ & 0.01 & 64 & Random \\
GG vs. AG/AA & 7 & 6209 & 5039 & $0.76(0.63,0.92)$ & 0.005 & 0.34 & 11 & Fixed \\
\hline
\end{tabular}

$\mathrm{OR}$, odds ratio; $\mathrm{Cl}$, confidence interval.

\section{Subgroup analysis}

Subgroup analyses by anatomic location, pathological subtype, ethnicity, source of controls, sample size $(\leq 1000$ and $>1000$ subjects), quality score (score $>7$ or $\leq 7$ ), adjustment and statistical power $(\geq 0.8$ or $<0.8)$ were performed only under the allele model, which had the largest sample size among all genotypic models (Table 2). Subgroup analyses indicated that the G allele at rs4072037 was associated with decreased gastric cancer risk regardless of anatomic location and pathological subtype. The association was significant in cardia gastric cancer $(\mathrm{OR}=0.75$, 95\% CI: 0.62-0.91), non-cardia gastric cancer $(\mathrm{OR}=0.66$, 95\% CI: 0.58-0.74), diffuse-type gastric cancer (OR=0.65, 95\% CI: 0.57-0.73) and intestinal-type gastric cancer (OR=0.75, 95\% CI: 0.66-0.83) (Additional file 3). Similarly, stratification by ample size, quality score, statistical power and source of control did not alter the results. There was also no difference between adjusted and unadjusted results. Moreover, we have noticed that heterogeneity could be reduced significantly when stratified by pathological subtype, quality score and control source, and statistical power, which may partly explain the source of heterogeneity.

Subgroup analyses were also performed according to ethnicity. The results indicated that $\mathrm{G}$ allele was associated with decreased gastric cancer risk in Asian ( $O R=0.69$, 95\% CI: 0.57-0.73) rather than Caucasian (OR=0.76, 95\% CI: 0.56-1.04). Stratification of the Asian subgroup into
Chinese, Japanese and Korean did not alter the results. Notably, the subgroup of Caucasian included fewer than three cohorts, which could not yield reliable results in the meta-analysis (Liberati et al. 2009).

\section{Sensitivity analysis}

One cohort was excluded at each time to investigate the influence of the individual data set on the overall results. The statistical significance of the overall results was not altered when any single study was excluded, confirming the stability of the results. However, $P$ for $Q$ test could reduce significantly when excluding the study of Li et al. (Li et al. 2013) and Song et al. (Song et al. 2013).

\section{Publication bias}

Begg's funnel plots (Figure 3) were conducted to assess publication bias for reported comparisons of rs4072037 and association with gastric cancer. The funnel plots were all symmetrical, indicating that there was no publication bias in the studies of MUC1 polymorphism and gastric cancer association (Begg's test: $p=0.115$, Egger's test: $p=0.060)$.

\section{Discussion}

The identification of genetic variants capable of modulating cancer development could be helpful for the early detection and design of targeted treatment and

\begin{tabular}{|c|c|c|c|c|c|c|}
\hline \multirow{2}{*}{$\begin{array}{l}\text { Study or Subgroup } \\
\text { Abnet } 2011\end{array}$} & \multirow{2}{*}{$\begin{array}{r}\text { log[Odds Ratio] } \\
-0.3425\end{array}$} & SE & Weight & \multirow{2}{*}{$\begin{array}{l}\begin{array}{c}\text { Odds Ratio } \\
\text { IV, Random, } 95 \% \mathrm{Cl}\end{array} \\
0.71[0.62,0.81]\end{array}$} & \multicolumn{2}{|c|}{$\begin{array}{c}\text { Odds Ratio } \\
\text { IV, Random, } 95 \% \mathrm{CI}\end{array}$} \\
\hline & & 0.0692 & $13.3 \%$ & & $\longrightarrow$ & \\
\hline Jia 2010 & -0.4173 & 0.1132 & $8.6 \%$ & $0.66[0.53,0.82]$ & & \\
\hline Li 2013 & -0.6733 & 0.1582 & $5.5 \%$ & $0.51[0.37,0.70]$ & & \\
\hline Palmer 2012 & -0.1075 & 0.1275 & $7.4 \%$ & $0.90[0.70,1.15]$ & & - \\
\hline Sadki 2011, Tokyo DGC & -0.4824 & 0.1045 & $9.3 \%$ & $0.62[0.50,0.76]$ & & \\
\hline Saeki 2011, Aichi & -0.5247 & 0.1458 & $6.2 \%$ & $0.59[0.44,0.79]$ & & \\
\hline Saeki 2011, Korea & -0.5539 & 0.1647 & $5.2 \%$ & $0.57[0.42,0.79]$ & & \\
\hline Saeki 2011, Tokyo ICC & -0.207 & 0.0955 & $10.2 \%$ & $0.81[0.67,0.98]$ & & \\
\hline Song 2013 & -0.1985 & 0.0664 & $13.7 \%$ & $0.82[0.72,0.93]$ & & \\
\hline Xu 2009 & -0.5075 & 0.2429 & $2.8 \%$ & $0.60[0.37,0.97]$ & & \\
\hline Yang 2012 & -0.4463 & 0.1912 & $4.1 \%$ & $0.64[0.44,0.93]$ & & \\
\hline Zhang 2011 & -0.3285 & 0.0681 & $13.5 \%$ & $0.72[0.63,0.82]$ & $\longrightarrow$ & \\
\hline Total $(95 \% \mathrm{Cl})$ & & & $100.0 \%$ & $0.70[0.64,0.76]$ & & \\
\hline \multicolumn{5}{|c|}{$\begin{array}{l}\text { Heterogeneity: } \text { Tau }^{2}=0.01 ; \mathrm{Chi}^{2}=20.62, \mathrm{df}=11(\mathrm{P}=0.04) ; \mathrm{I}^{2}=47 \% \\
\text { Test for overall effect: } \mathrm{Z}=8.20(\mathrm{P}<0.00001)\end{array}$} & $\begin{array}{ll}1 & 1 \\
0.5 & 0.7\end{array}$ & 1.5 \\
\hline
\end{tabular}

Figure 2 Forest plot describing MUC1 rs 4072037 and susceptibility to gastric cancer under the allele model. The horizontal lines represent $95 \% \mathrm{Cls}$ for estimating the outcome of the $\mathrm{G}$ allele versus the A allele in the meta-analysis. ( $\mathbf{\square})$ Overall estimates of the effects. Cl, confidence interval; OR, odds ratio. 
Table 2 Subgroup meta-analyses of the association between MUC1 polymorphism rs4072037 and gastric cancer risk under the allele model

\begin{tabular}{|c|c|c|c|c|c|c|c|c|}
\hline Comparison & No. of datasets & Case & Control & OR $(95 \% \mathrm{Cl})$ & $P$ for OR & $P$ for heterogeneity & $I^{2}(\%)$ & Model \\
\hline Overall & 12 & 10410 & 11437 & $0.70(0.64,0.76)$ & $<0.00001$ & 0.04 & 47 & Random \\
\hline \multicolumn{9}{|l|}{ Anatomic location } \\
\hline Cardia gastric cancer & 5 & 2362 & 7426 & $0.75(0.62,0.91)$ & 0.004 & 0.04 & 63 & Random \\
\hline Non-cardia gastric caner & 5 & 5161 & 7426 & $0.66(0.58,0.74)$ & $<0.00001$ & 0.15 & 43 & Fixed \\
\hline \multicolumn{9}{|l|}{ Pathological subtype } \\
\hline Diffuse-type gastric cancer & 6 & 1756 & 5191 & $0.65(0.57,0.73)$ & $<0.00001$ & 0.29 & 19 & Fixed \\
\hline Intestinal-type gastric cancer & 4 & 1727 & 3354 & $0.75(0.66,0.83)$ & $<0.00001$ & 0.48 & 0 & Fixed \\
\hline \multicolumn{9}{|l|}{ Ethnicity } \\
\hline Asian & 10 & 9826 & 10853 & $0.69(0.63,0.75)$ & $<0.00001$ & 0.05 & 47 & Random \\
\hline Caucasion & 2 & 584 & 584 & $0.76(0.56,1.04)$ & 0.08 & 0.07 & 70 & Random \\
\hline \multicolumn{9}{|l|}{ Sample size } \\
\hline$>1000$ & 6 & 8652 & 9614 & $0.73(0.69,0.78)$ & $<0.00001$ & 0.10 & 45 & Fixed \\
\hline$\leq 1000$ & 6 & 1758 & 1823 & $0.66(0.59,0.75)$ & $<0.00001$ & 0.10 & 47 & Fixed \\
\hline \multicolumn{9}{|l|}{ Quality score } \\
\hline$>7$ & 5 & 6183 & 5742 & $0.73(0.65,0.82)$ & $<0.00001$ & 0.27 & 23 & Fixed \\
\hline$\leq 7$ & 7 & 4227 & 5695 & $0.69(0.64,0.75)$ & $<0.00001$ & 0.22 & 28 & Fixed \\
\hline \multicolumn{9}{|l|}{ Control source } \\
\hline Hospital-based & 7 & 2683 & 3958 & $0.65(0.59,0.72)$ & $<0.00001$ & 0.16 & 35 & Fixed \\
\hline Population-based & 5 & 7727 & 7479 & $0.75(0.70,0.81)$ & $<0.00001$ & 0.19 & 34 & Fixed \\
\hline \multicolumn{9}{|l|}{ Adjustment } \\
\hline Yes & 9 & 9688 & 10612 & $0.69(0.63,0.76)$ & $<0.00001$ & 0.04 & 51 & Random \\
\hline No & 3 & 722 & 825 & $0.74(0.63,0.86)$ & 0.0001 & 0.13 & 51 & Fixed \\
\hline \multicolumn{9}{|l|}{ Statistical power } \\
\hline$\geq 0.8$ & 6 & 5595 & 8620 & $0.67(0.62,0.72)$ & $<0.00001$ & 0.22 & 0.29 & Fixed \\
\hline$<0.8$ & 6 & 4815 & 4081 & $0.78(0.72,0.85)$ & $<0.00001$ & 0.27 & 0.22 & Fixed \\
\hline
\end{tabular}

$\mathrm{OR}$, odds ratio; $\mathrm{Cl}$, confidence interval.

prevention strategies. With high interest in gene susceptibility to carcinogenesis, increasing efforts have been devoted to the study of genetic variants and gastric cancer risk. Since $M U C 1$ polymorphism has been reported to be associated with gastric cancer, several studies were performed to validate this finding. However, the results were inconsistent especially in different pathological type. Therefore, we performed the first meta-analysis to assess the relationship between MUC1 gene polymorphism rs4072037 and gastric cancer susceptibility.

The meta-analysis included 10,410 gastric cancer patients and 11,437 controls, which was far larger than the sample size of the discovery study and the GWAS. Association of MUC1 rs4072037 and gastric cancer susceptibility was detected under all genotypic models without significant heterogeneity, suggesting decreased gastric cancer risk for individuals carrying the $G$ allele. Although some of the included studies did not have enough statistical power, this did not influence the overall results and confidence because in the subgroup analyses, studies with power larger than or less than 0.8 yielded similar results with statistical significance.

A large-sample-size Japanese study (Saeki et al. 2011) demonstrated that MUC1 rs4072037 was associated with gastric cancer risk in diffuse-type gastric cancer rather than intestinal-type gastric cancer. However, our metaanalysis detected significant association in both subtypes. The sample size of the intestinal-type gastric cancer subgroup in our meta-analysis was twice larger than that in the Japanese study, indicating that our result was solid.

Subgroup analyses also revealed that $G$ allele at rs4072037 was associated with gastric cancer risk in Asian rather than Caucasian. Interestingly, we notice that the allele frequencies of $\mathrm{G}$ allele were more than 0.5 in both the Caucasian studies, while $\mathrm{G}$ was the minor allele in all the Asian studies. This remarkable difference in the frequency of the $\mathrm{G}$ allele might be due to distinct genetic backgrounds of different ethnicities and this might attribute to the different susceptibility of $G$ allele 


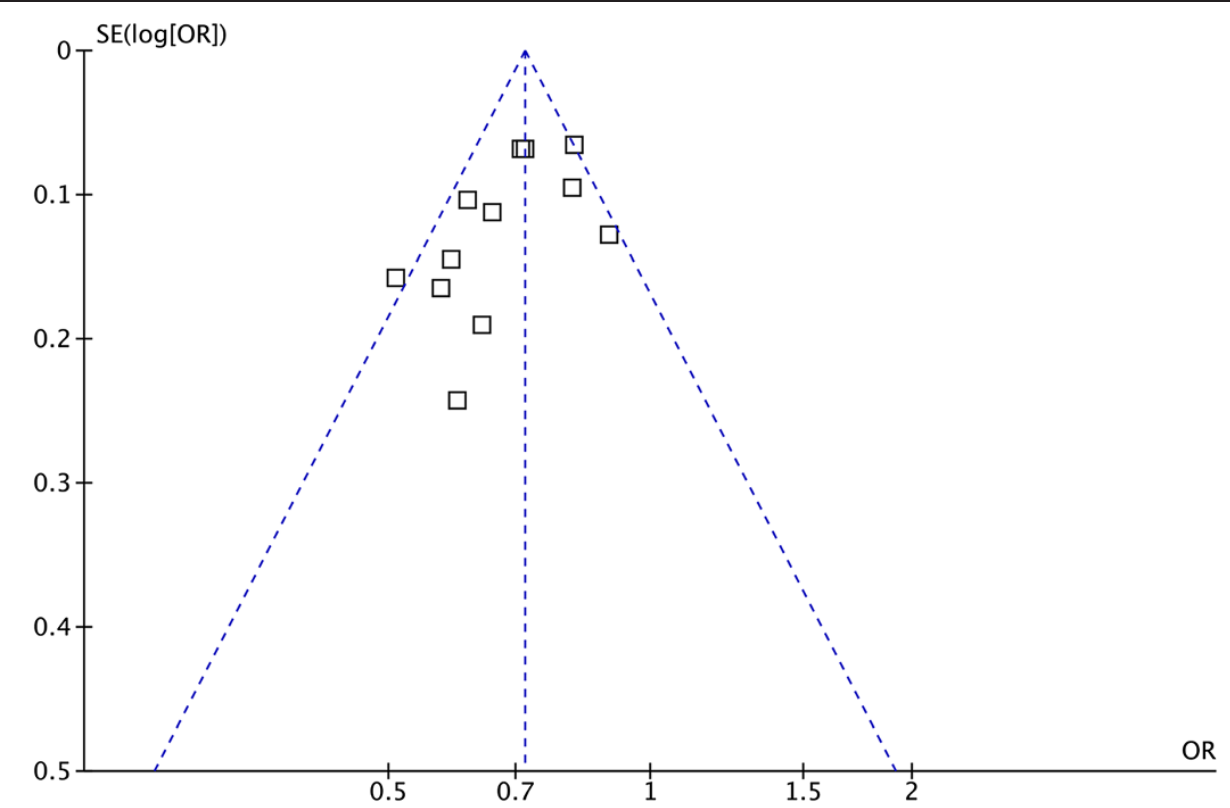

Figure 3 Funnel plot of the association between MUC1 rs4072037 and susceptibility to gastric cancer under the allele model. OR, odds ratio; SE, standard error.

to gastric cancer. Further large studies of multiethnic groups are needed before a comprehensive conclusion could be made.

MUC1 is a highly polymorphic transmembrane glycoprotein expressed on the surface of many epithelia, including gastric mucosa. It acts as a barrier against exogenous insults in normal epithelial cells. In contrast, once the cells lose cell polarity, MUC1 protein interacts freely with other molecules including membrane receptors involved in cell growth and, consequently, promotes cell growth and acts for tumorigenesis (Kufe 2009). Interestingly, SNP rs4072037, located in exon 2 of MUC1 gene, controls alternative splicing of the 5'-exon 2 region, resulting in both full-length transcripts and those lacking the polymorphic tandem repeat domain (Ng et al. 2008). The different protein products encoded by the two splice variants differ in the protective function of gastric mucosa (Saeki et al. 2011), which ultimately results in the difference in GC susceptibility. Therefore, our meta-analysis provided additional data supporting the potential functional role of $M U C 1$ in gastric cancer carcinogenesis, which needs to be authenticated through molecular and cellular approaches.

There is a recently published meta-analysis on $M U C 1$ rs4072037 and the risk of gastric cancer performed by Zheng et al. (Zheng et al. 2013) and we believe our study has some advantages over theirs' for the following reasons. First, Zhang et al. (Zheng et al. 2013) falsely calculated the number of total controls in their manuscript. In one of the included studies conducted by Seaki et al. (Saeki et al. 2011), the "Diffuse, Tokyo data set" and
"Intestinal, Tokyo data set" (referred to as data set "a" and " $\mathrm{d}$ " in Zheng et al's manuscript) shared the same group of controls, thus the total number of controls should be 9,060 instead of 10,324 in the meta-analysis. Second, the breakdown of the patient groups as independent datasets in one study in the overall analyses may introduce analysis bias. This method should be used only if the combined data is not available. It is understandable that the study by Seaki et al. (Saeki et al. 2011) only reported the separate results in different ethnic groups instead of combined results, which is also regarded as independent datasets in our meta-analysis. However, the patients in the study by Abnet et al. (Abnet et al. 2010) should not be separated as combined data was also reported. Third, we have concluded that MUC1 rs4072037 was associated with gastric cancer risk in Asian $(\mathrm{OR}=0.69$, 95\% CI: 0.63-0.75) rather than Caucasian (OR=0.76, 95\% CI: 0.56-1.04), which is different from the conclusion of Zheng et al. (Zheng et al. 2013). We find that Zheng et al. (Zheng et al. 2013) may have drawn a wrong conclusion by adopting the fixed effect model with the $I^{2}$ of $70 \%$ and $P$ for Q-test of 0.07 , while a random effect model should be used. Given these limitations and errors of the metaanalysis conducted by Zheng et al. (Zheng et al. 2013), their conclusions should be adopted with caution. Moreover, we have done a more profound literature search, and included three more studies (Yang et al. 2012) (Li et al. 2013) compared with the paper by Zheng et al. (Zheng et al. 2013) and our subgroup analyses were more detailed than that of Zheng et al. (Zheng et al. 2013). Besides all the parameters in Zheng et al's paper (Zheng et al. 2013), 
we also included source of control, adjustment, sample size and quality in the subgroup analyses.

However, some limitations of our meta-analysis should be acknowledged when interpreting the results. First, we were unable to conduct stratified analyses based on possible confounders such as age, $H$. pylori infection, smoking status, and alcohol intake due to insufficient data. Second, the data for Caucasian was limited. Third, the breakdown of the patient groups as independent datasets in one study (Saeki et al. 2011) in the overall analyses may introduce analysis bias. However, this study only reported the separate results in different ethnic groups instead of combined results. Under this circumstance, although it is recommended that tests for funnel plot asymmetry should be used only when there are at least 10 studies included in the meta-analysis (Ioannidis and Trikalinos 2007), we still tested the publication bias because we have 12 datasets in 9 studies. In addition, it is not mentioned whether the cancer-free hospital-based controls were gastritis patients, which limits our analyses on the role of MUC1 rs4072037 in gastric carcinogenesis when compared with the population-based healthy controls.

In conclusion, our meta-analysis summarizes the existing data on $M U C 1$ polymorphism rs4072037 and gastric cancer susceptibility. The results reveal that the presence of the $\mathrm{G}$ allele contributes to protection against gastric cancer in Asian, regardless of anatomic location and pathological subtype. Further large studies of multiethnic groups and investigation of confounders are warranted to validate these findings.

\section{Additional files}

\section{Additional file 1: PRISMA 2009 checklist. \\ Additional file 2: Characteristics of included studies.}

Additional file 3: Forest plots describing subgroup analyses of MUC1 rs4072037 and susceptibility to gastric cancer under the allele model.

\section{Competing interests}

The authors declare that they have no competing interest.

\section{Authors' contributions}

$L X Y, W Z C, Z X W$ and $L J$ were responsible for conception and study design. LXY, WZC and ZXW participated in literature search, study selection and acquisition of data. LXY, WZC, CJJ, TWB, GL and WZ contributed to data analysis and interpretation. $\amalg$ participated in the coordination and revised the manuscript. All authors read and approved the final manuscript.

\section{Acknowledgments}

The authors gratefully acknowledge Ms. Mary Smith for her kind help in language editing.

\section{Author details}

'Department of Medical Oncology, Fudan University Shanghai Cancer Center; Department of Oncology, Shanghai Medical College, Fudan University, Shanghai 200032, PR China. 'Liver Cancer Institute, Zhongshan Hospital, Fudan University, Shanghai 200032, China.
Received: 4 August 2014 Accepted: 6 October 2014

Published: 13 October 2014

\section{References}

Abnet CC, Freedman ND, Hu N, Wang Z, Yu K, Shu XO, Yuan JM, Zheng W, Dawsey SM, Dong LM, Lee MP, Ding T, Qiao YL, Gao YT, Koh WP, Xiang YB, Tang ZZ, Fan JH, Wang C, Wheeler W, Gail MH, Yeager M, Yuenger J, Hutchinson A, Jacobs KB, Giffen CA, Burdett L, Fraumeni JF Jr, Tucker MA, Chow WH et al (2010) A shared susceptibility locus in PLCE1 at 10q23 for gastric adenocarcinoma and esophageal squamous cell carcinoma. Nat Genet 42(9):764-767, doi:10.1038/ng.649

Blaser MJ (2000) Linking Helicobacter pylori to gastric cancer. Nat Med 6(4):376-377, doi:10.1038/74627

Clark MF, Baudouin SV (2006) A systematic review of the quality of genetic association studies in human sepsis. Intensive Care Med 32(11):1706-1712, doi:10.1007/s00134-006-0327-y

DerSimonian R, Laird N (1986) Meta-analysis in clinical trials. Control Clin Trials 7(3):177-188

Egger M, Davey Smith G, Schneider M, Minder C (1997) Bias in meta-analysis detected by a simple, graphical test. BMJ (Clin Res Ed) 315(7109):629-634

Gendler SJ (2001) MUC1, the renaissance molecule. J Mammary Gland Biol Neoplasia 6(3):339-353

loannidis JP, Trikalinos TA (2007) The appropriateness of asymmetry tests for publication bias in meta-analyses: a large survey. CMAJ 176(8):1091-1096, doi:10.1503/cmaj.060410

Jemal A, Bray F, Center MM, Ferlay J, Ward E, Forman D (2011) Global cancer statistics. CA Cancer J Clin 61(2):69-90, doi:10.3322/caac.20107

Jia Y, Persson C, Hou L, Zheng Z, Yeager M, Lissowska J, Chanock SJ, Chow WH, Ye W (2010) A comprehensive analysis of common genetic variation in MUC1, MUC5AC, MUC6 genes and risk of stomach cancer. Cancer Causes Control 21(2):313-321, doi:10.1007/s10552-009-9463-3

Kufe DW (2009) Mucins in cancer: function, prognosis and therapy. Nat Rev Cancer 9(12):874-885, doi:10.1038/nrc2761

Li M, Huang L, Qiu H, Fu Q, Li W, Yu Q, Sun L, Zhang L, Hu G, Hu J, Yuan X (2013) Helicobacter pylori Infection Synergizes with Three InflammationRelated Genetic Variants in the GWASs to Increase Risk of Gastric Cancer in a Chinese Population. PLoS One 8(9):e74976, doi:10.1371/journal.pone.0074976

Liberati A, Altman DG, Tetzlaff J, Mulrow C, Gotzsche PC, loannidis JP, Clarke M, Devereaux PJ, Kleijnen J, Moher D (2009) The PRISMA statement for reporting systematic reviews and meta-analyses of studies that evaluate health care interventions: explanation and elaboration. Ann Intern Med 151(4):W65-94

Linden SK, Sheng YH, Every AL, Miles KM, Skoog EC, Florin TH, Sutton P, McGuckin MA (2009) MUC1 limits Helicobacter pylori infection both by steric hindrance and by acting as a releasable decoy. PLoS Pathog 5(10):e1000617, doi:10.1371/journal.ppat.1000617

Mantel N, Haenszel W (1959) Statistical aspects of the analysis of data from retrospective studies of disease. J Natl Cancer Inst 22(4):719-748

Ng W, Loh AX, Teixeira AS, Pereira SP, Swallow DM (2008) Genetic regulation of MUC1 alternative splicing in human tissues. Br J Cancer 99(6):978-985, doi:10.1038/sj.bjc.6604617

Palmer AJ, Lochhead P, Hold GL, Rabkin CS, Chow WH, Lissowska J, Vaughan TL, Berry S, Gammon M, Risch H, El-Omar EM (2012) Genetic variation in C20orf54, PLCE1 and MUC1 and the risk of upper gastrointestinal cancers in Caucasian populations. Eur J Cancer Prev 21(6):541-544, doi:10.1097/ CEJ.0b013e3283529b79

Saeki N, Saito A, Choi IJ, Matsuo K, Ohnami S, Totsuka H, Chiku S, Kuchiba A, Lee YS, Yoon KA, Kook MC, Park SR, Kim YW, Tanaka H, Tajima K, Hirose H, Tanioka F, Matsuno Y, Sugimura H, Kato S, Nakamura T, Nishina T, Yasui W, Aoyagi K, Sasaki H, Yanagihara K, Katai H, Shimoda T, Yoshida T, Nakamura Y et al (2011) A functional single nucleotide polymorphism in mucin 1, at chromosome 1q22, determines susceptibility to diffuse-type gastric cancer. Gastroenterology 140(3):892-902, doi:10.1053/j.gastro.2010.10.058

Schlesselman J (1982) Case - Control Studies, Design, Conduct, Analysis. Oxford University Press, Oxford, pp 220-226

Skoog EC, Sjoling A, Navabi N, Holgersson J, Lundin SB, Linden SK (2012) Human gastric mucins differently regulate Helicobacter pylori proliferation, gene expression and interactions with host cells. PLoS One 7(5):e36378, doi:10.1371/journal.pone.0036378

Song HR, Kim HN, Kweon SS, Choi JS, Shim HJ, Cho SH, Chung IJ, Park YK, Kim SH, Choi YD, Joo KW, Shin MH (2013) Common genetic variants at 1q22 and 10q23 
and gastric cancer susceptibility in a Korean population. Tumour Biol, doi:10.1007/s13277-013-1409-4

Wu MS, Chen CJ, Lin JT (2005) Host-environment interactions: their impact on progression from gastric inflammation to carcinogenesis and on development of new approaches to prevent and treat gastric cancer. Cancer Epidemiol Biomarkers Prev 14(8):1878-1882, doi:10.1158/1055-9965.epi-04-0792

Xu Q, Yuan Y, Sun LP, Gong YH, XU Y, Yu XW, Dong NN, Lin GD, Smith PN, Li RW (2009) Risk of gastric cancer is associated with the MUC1 568 A/G polymorphism. Int J Oncol 35(6):1313-1320

Yang XX, Li FX, Zhou CP, Hu NY, Wu YS, Li M (2012) Association of Genetic Polymorphisms at 1q22 but not 10q23 with Gastric Cancer in a Southern Chinese Population. Asian Pac J Cancer Prev 13(6):2519-2522, doi:10.7314/ apjcp.2012.13.6.2519

Zhang HZ, Jin G, Li H, Ren C, Ding Y, Zhang Q, Deng B, Wang J, Hu Z, Xu Y, Shen H (2011) Genetic variants at $1 \mathrm{q} 22$ and 10q23 reproducibly associated with gastric cancer susceptibility in a Chinese population. Carcinogenesis 32(6):848-852, doi:10.1093/carcin/bgr051

Zheng L, Zhu C, Gu J, Xi P, Du J, Jin G (2013) Functional polymorphism rs4072037 in MUC1 gene contributes to the susceptibility to gastric cancer: evidence from pooled 6,580 cases and 10,324 controls. Mol Biol Rep, doi:10.1007/s11033-013-2682-4

doi:10.1186/2193-1801-3-599

Cite this article as: Liu et al:: MUC1 gene polymorphism rs4072037 and susceptibility to gastric cancer: a meta-analysis. SpringerPlus 2014 3:599.

\section{Submit your manuscript to a SpringerOpen ${ }^{\circ}$ journal and benefit from:}

- Convenient online submission

- Rigorous peer review

- Immediate publication on acceptance

- Open access: articles freely available online

- High visibility within the field

- Retaining the copyright to your article

Submit your next manuscript at $\gg$ springeropen.com 